\title{
Angiopoietin-Like Protein 2 Induces Synovial Inflammation in the Facet Joint Leading to Degenerative Changes via Interleukin-6 Secretion
}

\author{
Kazuki Sugimoto ${ }^{1,}$, Takayuki Nakamura ${ }^{1, *}$, Takuya Tokunaga ${ }^{1}$, Yusuke Uehara ${ }^{1}$, \\ Tatsuya Okada ${ }^{1}$, Takuya Taniwaki ${ }^{1}$, Toru Fujimoto ${ }^{1}$, Yuichi Oike ${ }^{2}$, Eiichi Nakamura ${ }^{1}$ \\ ${ }^{1}$ Department of Orthopaedics, Graduate School of Medical Science, Kumamoto University, Kumamoto, Japan \\ ${ }^{2}$ Department of Molecular Genetics, Graduate School of Medical Science, Kumamoto University, Kumamoto, Japan
}

\begin{abstract}
Study Design: Experimental human study.
Purpose: To determine whether angiopoietin-like protein 2 (ANGPTL2) is highly expressed in the hyperplastic facet joint (FJ) synovium and whether it activates interleukin-6 (IL-6) secretion in FJ synoviocytes.

Overview of Literature: Mechanical stress-induced synovitis is partially, but significantly, responsible for degenerative and subsequently osteoarthritic changes in the FJ tissues in patients with lumbar spinal stenosis (LSS). However, the underlying molecular mechanism remains unclear. IL-6 is highly expressed in degenerative FJ synovial tissue and is responsible for local chronic inflammation. ANGPTL2, an inflammatory and mechanically induced mediator, promotes the expression of IL-6 in many cells.

Methods: FJ tissues were harvested from five patients who had undergone lumbar surgery. Immunohistochemistry for ANGPTL2, IL-6, and cell markers was performed in the FJ tissue samples. After cultured synoviocytes from the FJ tissues were subjected to mechanical stress, ANGPTL2 expression and secretion were measured quantitatively using real-time quantitative reverse-transcription-polymerase chain reaction and enzyme-linked immunosorbent assay (ELISA), respectively. Following ANGPTL2 administration in the FJ synoviocytes, anti-nuclear factor- $\mathrm{KB}$ (NF-KB) activation was investigated using immunocytochemistry, and IL-6 expression and secretion were assayed quantitatively with or without NF-KB inhibitor. Moreover, we assessed whether ANGPTL2-induced IL-6 modulates leucocyte recruitment in the degenerative process by focusing on the monocyte chemoattractant protein-1 (MCP-1) expression.

Results: ANGPTL2 and IL-6 were highly expressed in the hyperplastic FJ synovium samples. ANGPTL2 was co-expressed in both, fibroblast-like and macrophage-like synoviocytes. Further, the expression and secretion of ANGPTL2 in the FJ synoviocytes increased in response to stimulation by mechanical stretching. ANGPTL2 protein promoted the nuclear translocation of NF-KB and induced IL-6 expression and secretion in the FJ synoviocytes. This effect was reversed following treatment with NF-KB inhibitor. Furthermore, ANGPTL2-induced IL-6 upregulated the MCP-1 expression in the FJ synoviocytes.

Conclusions: Mechanical stress-induced ANGPTL2 promotes chronic inflammation in the FJ synovium by activating IL-6 secretion, leading to FJ degeneration and subsequent LSS.
\end{abstract}

Keywords: Facet joint; Synoviocytes; Lumbar spinal stenosis; Angiopoietin-like protein 2; Interleukin-6

Received Jul 17, 2018; Revised Sep 6, 2018; Accepted Sep 11, 2018

Corresponding author: Takayuki Nakamura

Department of Orthopaedics, Graduate School of Medical Science, Kumamoto University, 1-1-1 Honjo, Chuo-ku, Kumamoto-shi, Kumamoto, 860-8556, Japan

Tel: +81-96-373-5226, Fax: +81-96-373-5228, E-mail: takayuki-n@kuh.kumamoto-u.ac.jp

*These two authors contributed equally to this work. 


\section{Introduction}

Lumbar spinal stenosis (LSS) includes intervertebral disc (IVD) degeneration, facet joint osteoarthritis (FJ-OA), and ligamentum flavum hypertrophy (LFH) [1]. IVD degeneration is the primary event, and the biomechanical insufficiency induced via this change causes a transfer of backward stresses to the facet joints (FJs), resulting in degenerative changes in the FJ, further lumbar instability, and LFH formation [2-4]. The morphological changes that accompany FJ-OA, including bony proliferation and apophyseal malalignment, assist in narrowing the lumbar spinal canal and intervertebral foramen, causing low back pain, leg pain, and intermittent claudication via direct nerve compression [4].

The etiology of osteoarthritis (OA) is multifactorial and includes systemic and local biomechanical factors [5]. Mechanical compression is one of the factors responsible for the degeneration of the appendicular joints, such as the knee. Subsequently, synovitis occurs locally in the joints, similar to rheumatoid arthritis (RA) [5,6]. The overexpression of inflammatory mediators activates the progression of cartilage degeneration in the joints [7-9]. Some trials have shown that FJ degeneration leading to $\mathrm{OA}$ changes may be partly attributable to the chronic inflammation observed in FJ tissues and in limb joints [10-12]. Interleukin-6 (IL-6) is considered a key factor in chronic inflammation and tissue remodeling [13], and high concentrations of IL-6 can be detected in the synovium of LSS-FJ tissues [10,11]. Several studies have investigated the biomechanisms underlying FJ inflammation; however, the mechanism of IL- 6 upregulation remains unclear.

Angiopoietin-like protein 2 (ANGPTL2), an inflammatory mediator, accelerates the progression of various noninfectious inflammatory processes that are associated with RA [14], abdominal aortic aneurysms [15], obesityassociated metabolic abnormalities [16], and LFH [17,18]. ANGPTL2 secretion is promoted by various pathological conditions, such as hypoxia, malnutrition, endoplasmic reticulum stress [16], and mechanical stress [17]. ANGPTL2 regulates the expression of some inflammatory cytokines, including IL-6 $[16,18]$. We hypothesized that ANGPTL2 is expressed at high levels in the degenerative FJ tissues of LSS patients and that mechanical stressinduced ANGPTL2 accelerates FJ inflammation via the activation of IL-6 expression. Based on this hypothesis, the present study aimed to investigate whether ANGPTL2 contributes to the pathogenesis of FJ inflammation using the FJ synovium and synovial cells harvested from LSS patients.

\section{Materials and Methods}

\section{Patients and ethics}

The FJ synovium samples $(\mathrm{n}=5)$ used in this study were provided by patients whose FJs were diagnosed as hyperplastic and hydrarthrotic during preoperative imaging examinations. The study population comprised two men and three women (age, 54-73 years; mean age, 65.6 years) who underwent lumbar surgery at the L4/5 level for LSS at Kumamoto University Hospital between September 2015 and March 2016. The study was approved by the Kumamoto University Ethics Committee (no., 1303), and written informed consent was obtained from all the patients. The trial complied with the principles in the Declaration of Helsinki in 1975.

\section{Immunohistochemistry}

FJ synovium samples were fixed in $4 \%$ paraformaldehyde (PFA), embedded in paraffin, and sliced into $4-\mu \mathrm{m}$-thick sections. After pretreatment of the sections with Target Retrieval Solution (pH 9; Dako, Glostrup, Denmark), endogenous peroxidases were blocked using 3\% hydrogen peroxide in methanol for 20 minutes. The following primary antibodies were used: anti-human ANGPTL2 (rabbit polyclonal, 1:100, 12316-1-AP; Proteintech, Chicago, IL, USA), anti-vimentin (mouse monoclonal, 1:100, ab8069; Abcam, Cambridge, MA, USA), anti-CD68 (mouse monoclonal, 1:100, ab31630; Abcam), anti-CD15 (mouse monoclonal, 1:50, ab188610; Abcam), anti-CD45 (rabbit polyclonal, 1:100, ab10558; Abcam), anti-IL-6 (rabbit polyclonal, 1:400, ab6672; Abcam), and anti-monocyte chemoattractant protein-1 (MCP-1, rabbit polyclonal, 1:100, ab9669; Abcam). Following treatment with a peroxidase-labeled secondary antibody (Histofine Simple Stain Max PO; Nichirei, Tokyo, Japan), immunoreactivity was visualized using histofine 3,3'-diaminobenzine (Dojindo Molecular Technologies, Kumamoto, Japan). The sections were counterstained with hematoxylin and analyzed using an Olympus BX51 light microscope (Olympus, Tokyo, Japan). For double immunofluorescence staining, antiANGPTL2 (1:200), anti-vimentin (1:200), and anti-CD68 
(1:200) were used as the primary antibodies, and Alexa Fluor 488-labeled anti-rabbit immunoglobulin G (IgG, 1:500, ab150077; Abcam) and Alexa Fluor 594-labeled anti-mouse IgG (1:500, ab150116; Abcam) were used as secondary antibodies. The nuclei were counterstained with 4',6'-diamidino-2-phenylindole (DAPI).

\section{Isolation and culture of facet joint synoviocytes}

FJ tissue samples that were harvested from LSS patients were washed in physiological saline, minced, and incubated for 1 hour at $37^{\circ} \mathrm{C}$ in Dulbecco's modified Eagle's medium (DMEM; Gibco Life Technologies, Carlsbad, CA, USA) that contained $0.2 \%$ type I collagenase (Gibco Life Technologies) and 1\% penicillin-streptomycin (Gibco Life Technologies). The suspension was filtered using a 100- $\mu \mathrm{m}$ mesh cell strainer (Becton Dickinson and Co., Franklin Lakes, NJ, USA), and the cells were seeded into the wells of a 6-well plate (Becton Dickinson and Co.) containing DMEM with $10 \%$ fetal bovine serum (FBS, Gibco Life Technologies) and 1\% penicillin-streptomycin. The subsequent experiments were conducted using cells from primary to the third passage.

\section{Stimulation of facet joint synoviocytes via mechani- cal stretching}

FJ synoviocytes were seeded into a silicone chamber (STB-CH-10; Strex Inc., Osaka, Japan) at a density of $1 \times 10^{5}$ cells/chamber. The chamber was attached to a stretching apparatus (STB-140, Strex Inc.), and a cyclic uniaxial stretch (10\% elongation) was applied for 6 hours or 12 hours $\left(10\right.$ cycles/min; $37^{\circ} \mathrm{C}, 5 \% \mathrm{CO}_{2}$ ) [17]. After stimulation via stretching, total RNA was isolated from the cells and reverse-transcribed for analyzing ANGPTL2 expression using polymerase chain reaction (PCR). The relative abundance of target transcripts was normalized to the expression of $18 \mathrm{~S}$ ribosomal RNA ( $18 \mathrm{~S}$ rRNA). The primers used for this analysis were as follows: ANGPTL2 forward (5'-GCCACCAAGTGTCAGCCTCA-3') and reverse (5'-TGGACAGTACCAAACATCCAACATC-3') and $18 \mathrm{~S}$ rRNA forward (5'-TTTGCGAGTACTCAACACCAACATC-3') and reverse (5'-GAGCATATCTTCGGCCCACAC-3'). For the analysis of ANGPTL2 protein, subconfluent FJ synoviocytes cultured in a silicone chamber (STB-CH-10) were washed with phosphate-buffered saline (PBS), and the medium was changed to serum-free
DMEM. After 24 hours of stimulation (10\% elongation, 10 cycles $/ \mathrm{min} ; 37^{\circ} \mathrm{C}, 5 \% \mathrm{CO}_{2}$ ), the medium was harvested. The secreted ANGPTL2 protein was measured using an ANGPTL2 enzyme-linked immunosorbent assay (ELISA) kit (IBL, Fujioka, Japan) as per the manufacturer's instructions.

\section{Stimulation of facet joint synoviocytes with angio- poietin-like protein 2}

For immunofluorescent staining, FJ synoviocytes were seeded onto a 4-well culture slide (Becton Dickinson and Co.), cultured subconfluently, treated with recombinant ANGPTL2 protein $(5 \mu \mathrm{g} / \mathrm{mL})$, and incubated for 1 hour $\left(37^{\circ} \mathrm{C}, 5 \% \mathrm{CO}_{2}\right)$. The cells were then fixed with $4 \%$ PFA and treated with anti-nuclear factor- $\kappa \mathrm{B}$ (NFкB) p65 antibody (rabbit polyclonal, 1:100, sc-372; Santa Cruz Biotechnology, Santa Cruz, CA, USA). Alexa Fluor 488-labeled anti-rabbit IgG (1:500, ab150077; Abcam) was applied as the secondary antibody, and DAPI was used for nuclear staining.

ANGPTL2 protein $(5 \mu \mathrm{g} / \mathrm{mL})$ was added to the wells of a 12-well plate (Becton Dickinson and Co.) containing subconfluent FJ synoviocytes with or without the NF- $\kappa B$ inhibitor BAY 11-7082 (10 $\mu \mathrm{M}$; Wako Pure Chemical Industries, Osaka, Japan). DMEM, containing 10\% FBS and $1 \%$ penicillin-streptomycin, was added to each well, and the cells were incubated for 6 hours before RNA extraction. The IL6 mRNA expression was evaluated using realtime quantitative reverse-transcription (qRT)-PCR. The relative abundance of target transcripts was normalized to the expression of $18 \mathrm{~S}$ rRNA. The IL6 primers were as follows: forward, (5'-AAGCCAGAGCTGTGCAGATGAGTA-3') and reverse, (5'-TGTCCTGCAGCCACTGGTTC-3'). In order to analyze the IL-6 protein expression after ANGPTL2 administration, subconfluent FJ synoviocytes cultured in a 6-well plate were washed with PBS, and the medium was changed to serum-free DMEM. ANGPTL2 $(5 \mu \mathrm{g} / \mathrm{mL})$ was added to each well, plates were incubated for 24 hours $\left(37^{\circ} \mathrm{C}, 5 \% \mathrm{CO}_{2}\right)$, and the amount of secreted IL-6 protein was measured using IL-6 ELISA kit (R\&D Systems, Minneapolis, MN, USA).

\section{Evaluation of monocyte chemoattractant protein-1 expression}

To evaluate the MCP-1 response to inflammatory 
stimuli, recombinant IL-6 protein $(200 \mathrm{ng} / \mathrm{mL}$; Wako Pure Chemical Industries, Osaka, Japan) with the same amount of soluble IL-6 receptor a (sIL-6Ra; Wako Pure Chemical Industries) was first added to a 12-well plate (Becton Dickinson and Co.) to culture the synoviocytes. Thereafter, ANGPTL2 protein $(5 \mu \mathrm{g} / \mathrm{mL})$ was added to the same kind of plate (Becton Dickinson and Co.) containing subconfluent $\mathrm{FJ}$ synoviocytes with or without sIL-6Ra (200 ng/mL). Each plate was incubated for 6 hours before RNA extraction. MCP-1 mRNA expression was evaluated with qRT-PCR. The relative abundance of target transcripts was normalized to the expression of $18 \mathrm{~S}$ rRNA. The $M C P-1$ primers were as follows: forward, (5'-CAAACTGAAGCTCGCACTCTCGCC-3') and reverse, (5'-CTCCTAATGTCACGCACGATTT-3').

\section{Statistical analyses}

Data are expressed as the mean \pm standard error of the mean values. Student $t$-tests were used for performing
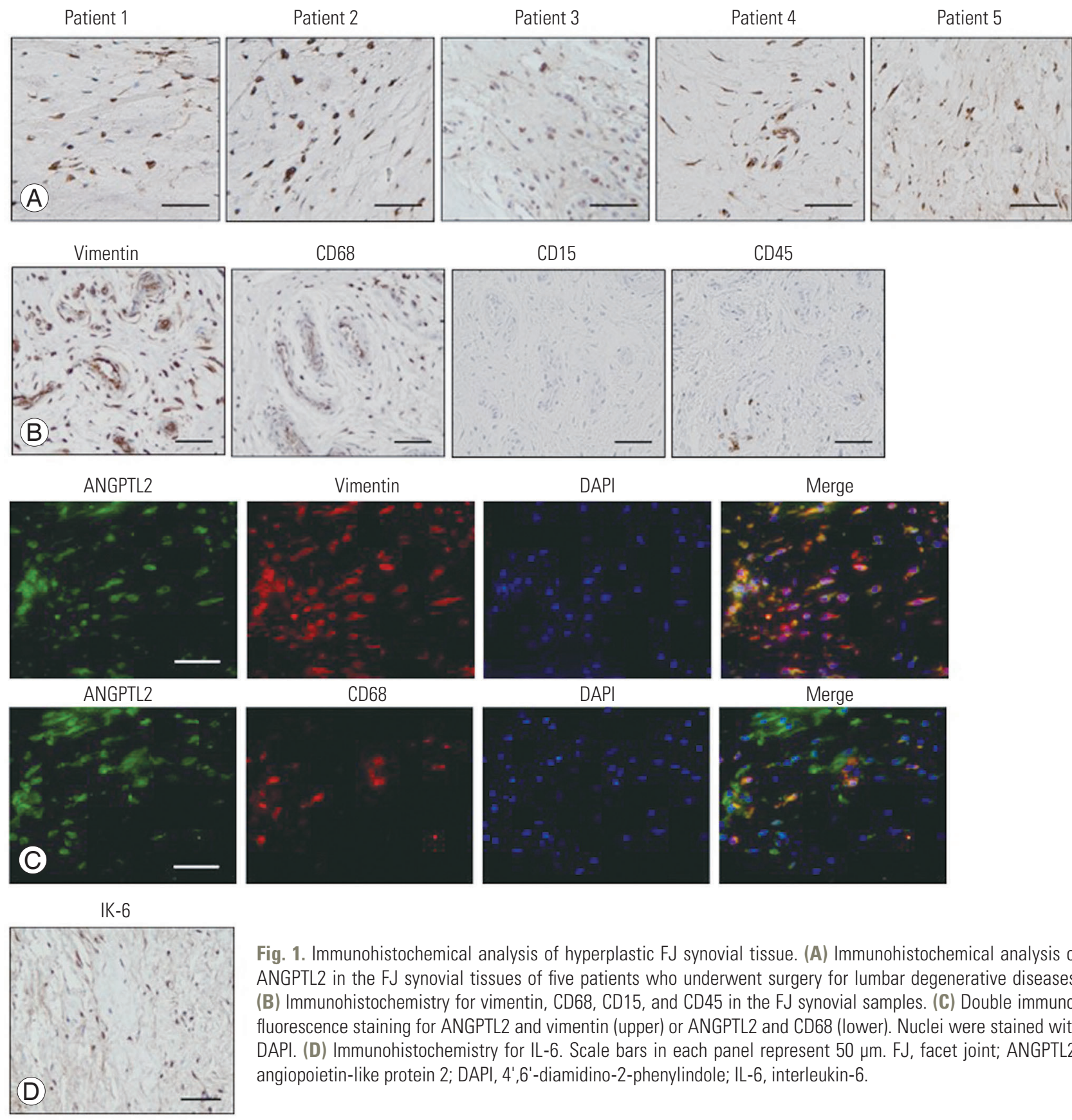

Fig. 1. Immunohistochemical analysis of hyperplastic FJ synovial tissue. (A) Immunohistochemical analysis of ANGPTL2 in the FJ synovial tissues of five patients who underwent surgery for lumbar degenerative diseases. (B) Immunohistochemistry for vimentin, CD68, CD15, and CD45 in the FJ synovial samples. (C) Double immunofluorescence staining for ANGPTL2 and vimentin (upper) or ANGPTL2 and CD68 (lower). Nuclei were stained with DAPI. (D) Immunohistochemistry for IL-6. Scale bars in each panel represent $50 \mu \mathrm{m}$. FJ, facet joint; ANGPTL2, angiopoietin-like protein 2; DAPI, 4',6'-diamidino-2-phenylindole; IL-6, interleukin-6. 
comparisons between the two groups in in vitro studies. All $p$-values $<0.05$ were considered to indicate significance. For statistical analyses, we performed post-hoc analysis using $\mathrm{G}^{\star}$ Power (ver. 3.1; Heinrich-Heine-Universität Düsseldorf, Düsseldorf, Germany; http://www.gpower.hhu.de/) and confirmed the statistical validity (power $>0.8$ ) for all tests [19].

\section{Results}

\section{Expression and cellular source of angiopoietin-like protein 2 in degenerative facet joint synovium}

A large number of ANGPTL2-expressing cells were identified in the hyperplastic FJ tissues in all LSS patients (Fig. 1A). Thereafter, the cell types present in the degenerative FJ tissues were examined with immunohistochemical analysis using antibodies against vimentin (a mesenchymal cell marker), CD68 (a macrophage marker), CD15 (a granulocyte marker), and CD45 (a lymphocyte marker). Patient cells expressed vimentin and CD68 at high levels, while the expression of CD15 and CD45 was very low, suggesting that hyperplastic FJ tissue mainly includes fibroblast-like synoviocytes and macrophagelike synoviocytes (Fig. 1B). Immunofluorescent double staining with anti-ANGPTL2 and anti-vimentin or antiCD68 antibodies showed that ANGPTL2 was partially expressed by vimentin-positive cells and CD68-positive cells. This suggests that ANGPTL2 is produced by both fibroblast-like and macrophage-like synoviocytes in the FJ tissues (Fig. 1C). IL-6 was also expressed in the cells of the hyperplastic FJ tissues (Fig. 1D), suggesting that IL-6 and ANGPTL2 are highly expressed in the degenerative FJ synovium.

\section{Upregulation of angiopoietin-like protein 2 by me-} chanical stretching stimulation

In order to determine whether mechanical stress directly induces ANGPTL2 expression, we subjected FJ synoviocytes isolated from LSS patients to repeated cyclic mechanical stretching, as described above. Stretching stimulation increased ANGPTL2 mRNA expression in the FJ synoviocytes and ANGPTL2 protein secretion in the culture medium (Fig. 2A, B). These findings suggest that ANGPTL2 production via mechanical stress could contribute to the pathological process underlying FJ degeneration.

\section{Induction of interleukin-6 by angiopoietin-like pro- tein 2 in facet joint synoviocytes}

ANGPTL2 promotes the nuclear translocation of NF- $\kappa \mathrm{B}$ and the subsequent induction of IL- $6[16,18]$; therefore, we investigated whether ANGPTL2 activates the nuclear translocation of NF- $\kappa \mathrm{B}$ and induces IL6 expression in FJ
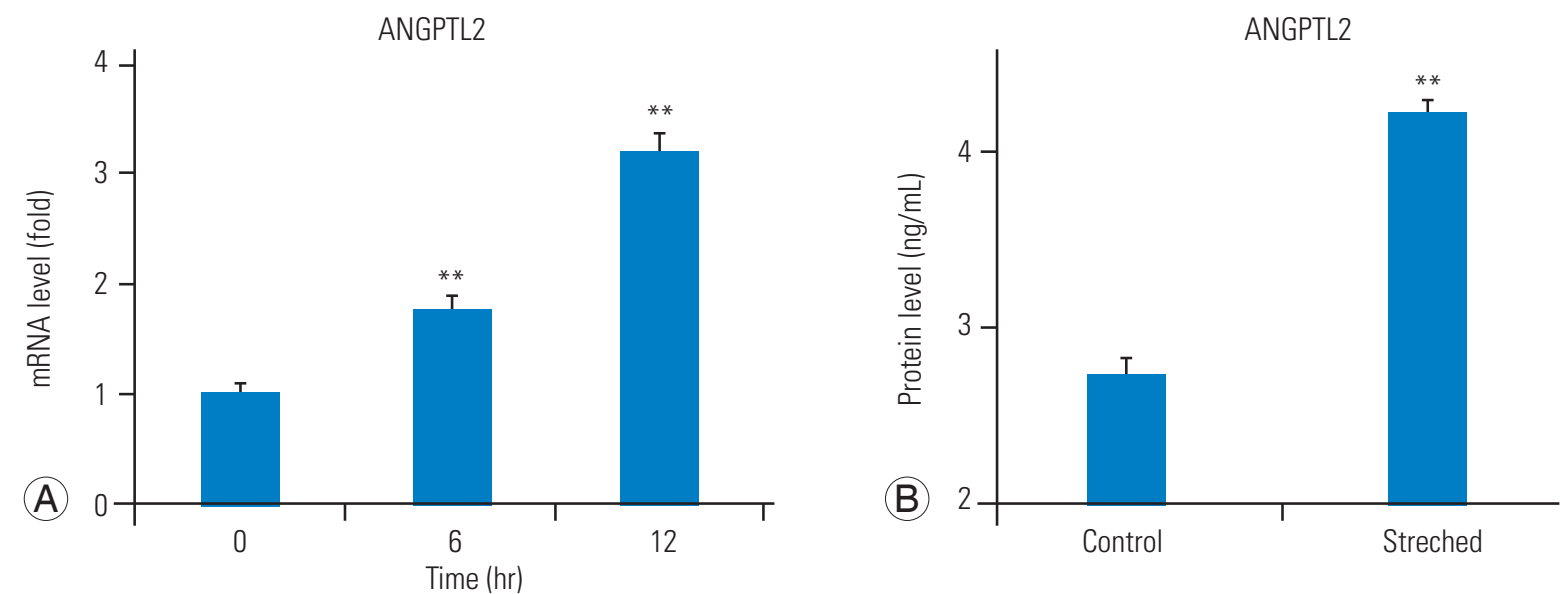

Fig. 2. ANGPTL2 expression and secretion are promoted by mechanical stretching stimulation in the FJ synoviocytes. (A) Changes in ANGPTL2 mRNA expression in the FJ synoviocytes ( $n=3$ ) after stretching (elongation ratio of $10 \%, 10$ cycles/min) for the indicated duration. ANGPTL2 expression in the FJ synoviocytes that were not subjected to stress (0 hr) was set to 1. (B) ANGPTL2 protein concentration in the culture medium of FJ synoviocytes ( $n=3$ ) after stretching (elongation ratio of 10\%, 10 cycles/min, $24 \mathrm{hr}$ ). Values are presented as mean \pm standard error of the mean values. Power $>0.8$ in the analyses. ANGPTL2, angiopoietin-like protein 2 ; FJ, facet joint. ${ }^{* *} p<0.01$ vs. the control. 


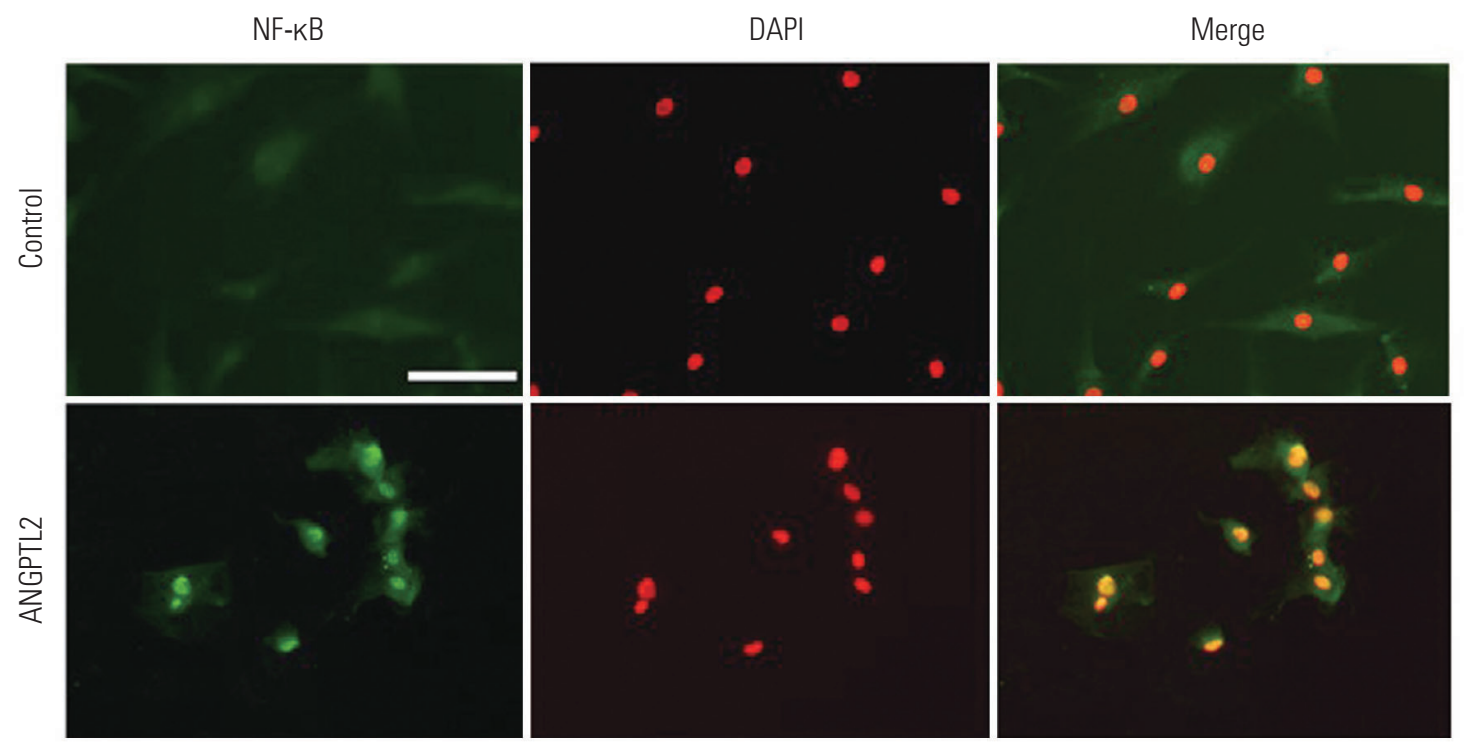

Fig. 3. Nuclear translocation of NF-KB is activated by ANGPTL2 in the FJ synoviocytes. Immunocytochemical analysis of nuclear translocation of NF-KB in the FJ synoviocytes following the administration of ANGPTL2 protein. The nuclei were stained with DAPI. The scale bar represents $50 \mu \mathrm{m}$. NF-kB, nuclear factor-kB; ANGPTL2, angiopoietin-like protein 2; FJ, facet joint; DAPI, 4',6'-diamidino-2-phenylindole.
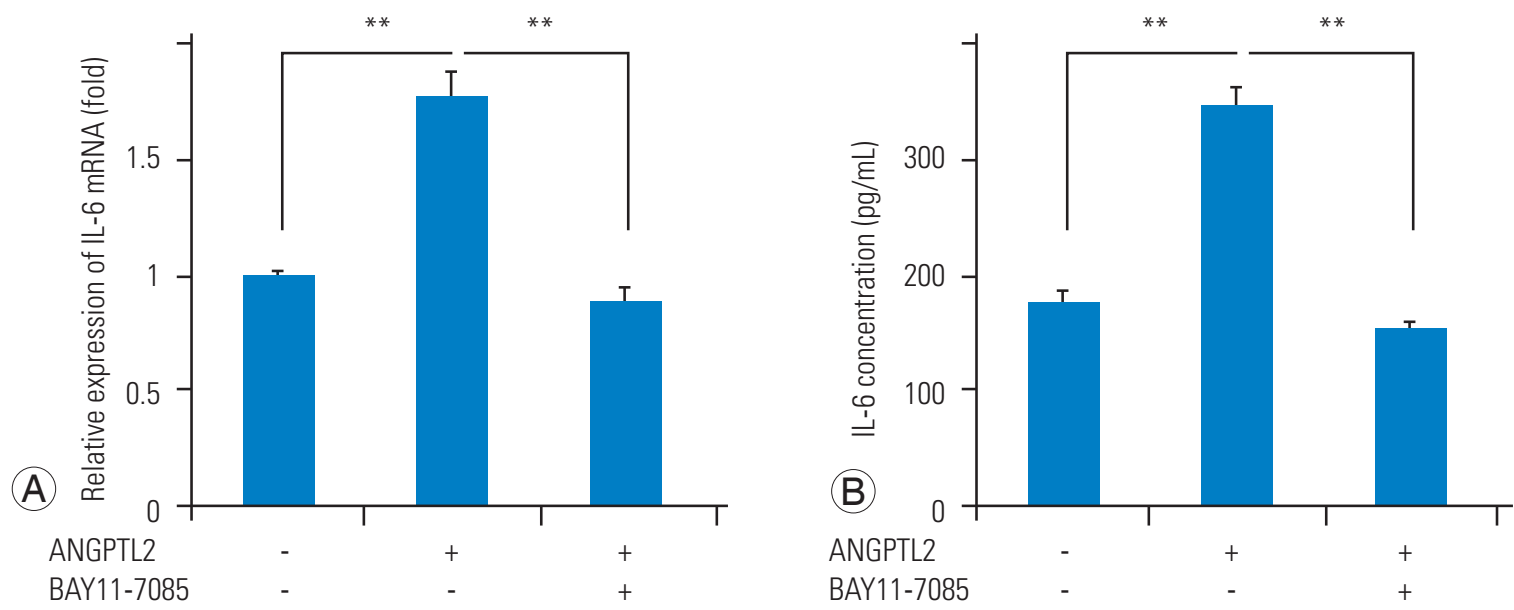

Fig. 4. IL-6 expression and secretion are upregulated by ANGPTL2 in the FJ synoviocytes. IL6 mRNA expression (A) and IL-6 secretion (B) in response to ANGPTL2 protein with or without treatment with BAY 11-7085 ( $n=3)$. The level of IL-6 expression in the controls (without ANGPTL2 treatment) was set to 1 . Values are presented as mean \pm standard error of the mean values. Power $>0.8$ in the analyses. IL-6, interleukin-6; ANGPTL2, angiopoietin-like protein 2; FJ, facet joint. ${ }^{* *} p<0.01$.

synoviocytes. We confirmed that NF- $\mathrm{kB}$ nuclear translocation in FJ synoviocytes was induced by ANGPTL2 treatment (Fig. 3). Further, ANGPTL2 upregulated the IL6 mRNA expression and IL-6 protein secretion in FJ synoviocytes. In contrast, NF- $\kappa \mathrm{B}$ inhibition via treatment with BAY11-7085 abrogated the elevation of IL- 6 at the mRNA and protein levels (Fig. 4A, B). These results demonstrate that ANGPTL2 induces IL-6 expression and secretion via the nuclear translocation of NF- $\kappa B$ in FJ synoviocytes.

\section{Induction of chronic inflammation by angiopoietin- like protein 2 in facet joint tissues}

As the combination of IL- 6 with sIL-6Ra increases the MCP-1 secretion of the stromal cells, favoring a transition from neutrophil to monocyte/macrophage accumulation and formulating chronic inflammation at the site of injury [13], we attempted to determine whether MCP-1 expresses in the LSS-FJ tissues and whether ANGPTL2-induced IL-6 upregulates the MCP-1 expression in FJ synovio- 

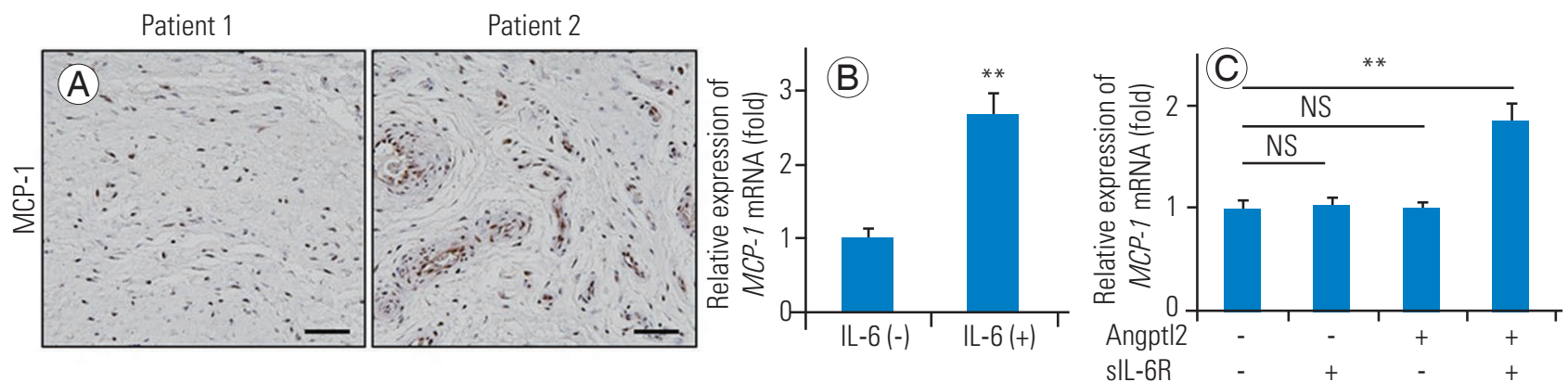

Fig. 5. MCP-1 is highly expressed in hyperplastic FJ tissues and the MCP-1 expression is promoted by ANGPTL2 in the FJ synoviocytes. (A) Immunohistochemical analysis of MCP-1 in the representative FJ synovial tissues taken from LSS patients. (B) Changes in the MCP-1 mRNA expression in the FJ synoviocytes ( $n=3$ ) after the $\mathrm{IL}-6 / \mathrm{slL}-6 \mathrm{R} \alpha$ administration. (C) Changes in the MCP-1 mRNA expression in the FJ synoviocytes ( $\mathrm{n}=3$ ) in response to ANGPTL2 protein with or without sIL-6Ro. The level of MCP-1 expression in the controls (without treatment) was set to 1. Values are presented as mean \pm standard error of the mean values. Power $>0.8$ in the analyses. MCP-1, monocyte chemoattractant protein-1; FJ, facet joint; ANGPTL2, angiopoietin-like protein 2; IL-6, interleukin-6; sIL-6R $\alpha$, soluble IL-6 receptor $\alpha$; NS, not significant. ${ }^{* *} p<0.01$ vs. the control.

cytes. As expected, several MCP-1-expressing cells were detected in the hyperplastic FJ tissues (Fig. 5A), and the expression of MCP-1 in FJ synoviocytes was elevated due to IL-6/sIL-6Ra administration (Fig. 5B). Only Angptl2 protein with sIL-6R $\alpha$ increased the $M C P-1$ mRNA expression (Fig. 5C). These findings suggest that ANGPTL2induced IL- 6 combined with sIL-6R $\alpha$ can raise the MCP1 secretion, leading to macrophage recruitment in $\mathrm{FJ}$ synovium and subsequent chronic inflammation.

\section{Discussion}

In this study, we showed that ANGPTL2 is abundantly expressed in the hyperplastic FJ tissues of LSS patients and that mechanical stress-induced ANGPTL2 upregulated the expression and secretion of IL-6 in the FJ synoviocytes via NF- $\mathrm{KB}$ activation and increased MCP-1 expression associated with chronic inflammation. We demonstrated that ANGPTL2 could be a key factor in promoting FJ inflammation by activating IL- 6 expression.

FJs have the same anatomy as the appendicular joints; they are covered by the hyaline cartilage and are enclosed by the synovium and capsule [2]. Similar to that in RA, local inflammatory reactions occur in the OA joints and many chemical factors derived from the synoviocytes participate in cartilage destruction [5-9]. An in vitro study reported that synoviocytes are potent sources of IL-6 that contributes to inflammatory arthropathies [20]. In clinical practice, IL-6 represents a significant biomarker of OA because the circulatory and intra-articular levels of IL-6 are increased in patients with $\mathrm{OA}$ of the knee. In particular, patients in the early stage of OA exhibit high serum IL-6 levels [21,22]. Some researchers have suggested that, similar to that in the limb joints, the first stage of OA changes in the FJs may result from repetitive mechanical stress, which subsequently induces FJ synovitis. They found that inflammatory cytokines, such as IL-6 were expressed at higher concentrations in the synovial membranes of LSS patients than in those of lumbar disc herniation (LDH) patients without severe FJ degeneration [10,11].

ANGPTL2 is induced under various stress conditions, such as hypoxia, malnutrition, endoplasmic reticulum stress [16], and mechanical stress [17]. In this study, the expression and secretion of ANGPTL2 from the synoviocytes of the hyperplastic FJ tissues were elevated in response to stretching stimulation. Therefore, the induction of ANGPTL2 by lumbar mechanical stress could partially trigger the first step of the FJ degenerative process. Based on the present results, we can conclude that ANGPTL2induced IL-6 can lead to synovitis that progresses to FJ inflammation and the subsequent degenerative process.

IL-6 plays important roles in regulating the transition from neutrophil (acute inflammation) to monocyte (chronic inflammation) recruitment and is a part of tissue remodeling and degenerative pathogenesis [13]. Our study found that there were few neutrophils and several macrophage-like cells in the degenerative FJ tissue, and that ANGPTL2 protein with sIL-6Ra elevated the MCP-1 expression in the cultured synoviocytes. Thus, our FJ samples were in the chronic inflammatory phase and ANGPTL2-induced IL-6 could recruit monocytes via MCP-1 and establish a chronic inflammatory condition. sIL-6Ra is derived from neutrophils in the acute inflammatory phase [13]; therefore, we consider that the neutrophils in 
the initial FJ inflammation might contribute to sIL-6Ra secretion and the serum receptor could induce MCP-1 in combination with IL-6 derived from the FJ synoviocytes, shifting to the FJ chronic inflammation. Further studies on FJ samples in the acute inflammatory phase are necessary to understand the mechanism.

Inflammation and degenerative changes in the FJs have been implicated in chronic lower back pain in about $25 \%$ of the patients [23]. Radiculopathy is induced not only by the direct nerve-root compression of the FJ osteophytes, but also by the spread of chemical factors, such as IL- 6 produced by the FJs [24-26]. Thus, our results indicate that radiculopathy and sciatica that are induced by chronic FJ inflammation, can also be treated by targeting ANGPTL2.

There are certain limitations of this study. First, our tissue sample size was very small because it was difficult to harvest tissue samples large enough for the histological analysis. Second, control FJ synovium specimens were unavailable because minimally invasive surgery has been adopted for $\mathrm{LDH}$ treatment at our institution, and LDHFJ tissues are too thin to harvest the samples without destroying the FJs and causing consequent iatrogenic lumbar instability. Therefore, a control group could not be employed, and the expression pattern of ANGPTL2 in normal FJ tissues was unclear. Further investigations using control samples are necessary for clarifying the degenerative mechanism.

\section{Conclusions}

Mechanical stress-induced ANGPTL2 produced by synoviocytes promotes FJ inflammation via the induction of IL- 6 secretion, resulting in degenerative changes and subsequent FJ-OA. ANGPTL2 could be a key mediator of FJ inflammation and degeneration; therefore, anti-ANGPTL2 treatment may be a novel strategy for preventing FJOA changes and subsequent LSS formation.

\section{Conflict of Interest}

No potential conflict of interest relevant to this article was reported.

\section{Author Contributions}

Conception and design: Kazuki Sugimoto, Takayuki Na- kamura; data acquisition: Kazuki Sugimoto; analysis of data: Kazuki Sugimoto, Takayuki Nakamura; drafting of the manuscript: Kazuki Sugimoto, Takayuki Nakamura; critical revision: Takayuki Nakamura; obtaining funding: Eiichi Nakamura; administrative support: Takuya Tokunaga, Yusuke Uehara, Tatsuya Okada, Takuya Taniwaki; and supervision: Toru Fujimoto, Yuichi Oike, Eiichi Nakamura.

\section{References}

1. Helms CA. CT of the lumbar spine: stenosis and arthrosis. Comput Radiol 1982;6:359-69.

2. Kalichman L, Hunter DJ. Lumbar facet joint osteoarthritis: a review. Semin Arthritis Rheum 2007;37:6980.

3. Yoshiiwa T, Miyazaki M, Notani N, Ishihara T, Kawano M, Tsumura H. Analysis of the relationship between ligamentum flavum thickening and lumbar segmental instability, disc degeneration, and facet joint osteoarthritis in lumbar spinal stenosis. Asian Spine J 2016;10:1132-40.

4. Kong $\mathrm{MH}$, Morishita $\mathrm{Y}, \mathrm{He} \mathrm{W}$, et al. Lumbar segmental mobility according to the grade of the disc, the facet joint, the muscle, and the ligament pathology by using kinetic magnetic resonance imaging. Spine (Phila Pa 1976) 2009;34:2537-44.

5. Felson DT, Zhang Y, Hannan MT, et al. Risk factors for incident radiographic knee osteoarthritis in the elderly: the Framingham Study. Arthritis Rheum 1997;40:728-33.

6. Smith MD, Triantafillou S, Parker A, Youssef PP, Coleman M. Synovial membrane inflammation and cytokine production in patients with early osteoarthritis. J Rheumatol 1997;24:365-71.

7. Pelletier JP, Martel-Pelletier J, Abramson SB. Osteoarthritis, an inflammatory disease: potential implication for the selection of new therapeutic targets. Arthritis Rheum 2001;44:1237-47.

8. Bonnet CS, Walsh DA. Osteoarthritis, angiogenesis and inflammation. Rheumatology (Oxford) 2005;44:7-16.

9. Loeser RF. Molecular mechanisms of cartilage destruction: mechanics, inflammatory mediators, and aging collide. Arthritis Rheum 2006;54:1357-60.

10. Igarashi A, Kikuchi S, Konno S, Olmarker K. Inflammatory cytokines released from the facet joint tissue 
in degenerative lumbar spinal disorders. Spine (Phila Pa 1976) 2004;29:2091-5.

11. Igarashi A, Kikuchi S, Konno S. Correlation between inflammatory cytokines released from the lumbar facet joint tissue and symptoms in degenerative lumbar spinal disorders. J Orthop Sci 2007;12:154-60.

12. Xu D, Sun Y, Bao G, et al. MMP-1 overexpression induced by IL-1 $\beta$ : possible mechanism for inflammation in degenerative lumbar facet joint. J Orthop Sci 2013;18:1012-9.

13. Gabay C. Interleukin- 6 and chronic inflammation. Arthritis Res Ther 2006;8 Suppl 2:S3.

14. Okada T, Tsukano H, Endo M, et al. Synoviocytederived angiopoietin-like protein 2 contributes to synovial chronic inflammation in rheumatoid arthritis. Am J Pathol 2010;176:2309-19.

15. Tazume H, Miyata K, Tian Z, et al. Macrophagederived angiopoietin-like protein 2 accelerates development of abdominal aortic aneurysm. Arterioscler Thromb Vasc Biol 2012;32:1400-9.

16. Tabata M, Kadomatsu T, Fukuhara S, et al. Angiopoietin-like protein 2 promotes chronic adipose tissue inflammation and obesity-related systemic insulin resistance. Cell Metab 2009;10:178-88.

17. Nakamura T, Okada T, Endo M, et al. Angiopoietinlike protein 2 induced by mechanical stress accelerates degeneration and hypertrophy of the ligamentum flavum in lumbar spinal canal stenosis. PLoS One 2014;9:e85542.

18. Nakamura T, Okada T, Endo M, Nakamura T, Oike Y, Mizuta $\mathrm{H}$. Angiopoietin-like protein 2 promotes inflammatory conditions in the ligamentum flavum in the pathogenesis of lumbar spinal canal stenosis by activating interleukin- 6 expression. Eur Spine J
2015;24:2001-9.

19. Faul F, Erdfelder E, Lang AG, Buchner A. G*Power 3: a flexible statistical power analysis program for the social, behavioral, and biomedical sciences. Behav Res Methods 2007;39:175-91.

20. Guerne PA, Zuraw BL, Vaughan JH, Carson DA, Lotz M. Synovium as a source of interleukin 6 in vitro: contribution to local and systemic manifestations of arthritis. J Clin Invest 1989;83:585-92.

21. Ding J, Niu X, Su Y, Li X. Expression of synovial fluid biomarkers in patients with knee osteoarthritis and meniscus injury. Exp Ther Med 2017;14:1609-13.

22. Livshits G, Zhai G, Hart DJ, et al. Interleukin-6 is a significant predictor of radiographic knee osteoarthritis: the Chingford Study. Arthritis Rheum 2009;60:2037-45.

23. Manchikanti L, Boswell MV, Singh V, Pampati V, Damron KS, Beyer CD. Prevalence of facet joint pain in chronic spinal pain of cervical, thoracic, and lumbar regions. BMC Musculoskelet Disord 2004;5:15.

24. Tachihara H, Kikuchi S, Konno S, Sekiguchi M. Does facet joint inflammation induce radiculopathy?: an investigation using a rat model of lumbar facet joint inflammation. Spine (Phila Pa 1976) 2007;32:406-12.

25. Ozaktay AC, Cavanaugh JM, Asik I, DeLeo JA, Weinstein JN. Dorsal root sensitivity to interleukin-1 beta, interleukin-6 and tumor necrosis factor in rats. Eur Spine J 2002;11:467-75.

26. Ozaktay AC, Kallakuri S, Takebayashi T, et al. Effects of interleukin-1 beta, interleukin-6, and tumor necrosis factor on sensitivity of dorsal root ganglion and peripheral receptive fields in rats. Eur Spine J 2006;15:1529-37. 